\title{
Optimised THz photoconductive devices based on low- temperature grown III-V compound semiconductors incorporating distributed Bragg reflectors
}

DOI:

10.1049/iet-opt.2016.0055

\section{Document Version}

Accepted author manuscript

Link to publication record in Manchester Research Explorer

Citation for published version (APA):

Wang, Y., Kostakis, I., Saeedkia, D., \& Missous, M. (2017). Optimised THz photoconductive devices based on lowtemperature grown III-V compound semiconductors incorporating distributed Bragg reflectors. IET Optoelectronics, 11(2), 53-57. https://doi.org/10.1049/iet-opt.2016.0055

\section{Published in:}

IET Optoelectronics

\section{Citing this paper}

Please note that where the full-text provided on Manchester Research Explorer is the Author Accepted Manuscript or Proof version this may differ from the final Published version. If citing, it is advised that you check and use the publisher's definitive version.

\section{General rights}

Copyright and moral rights for the publications made accessible in the Research Explorer are retained by the authors and/or other copyright owners and it is a condition of accessing publications that users recognise and abide by the legal requirements associated with these rights.

\section{Takedown policy}

If you believe that this document breaches copyright please refer to the University of Manchester's Takedown Procedures [http://man.ac.uk/04Y6Bo] or contact uml.scholarlycommunications@manchester.ac.uk providing relevant details, so we can investigate your claim.

\section{OPEN ACCESS}




\section{Optimized THz photoconductive devices based on low- temperature grown III-V compound semiconductors incorporating distributed Bragg reflectors}

\begin{tabular}{|r|l|}
\hline Journal: & IET Optoelectronics \\
\hline Manuscript ID & OPT-SI-2016-0055.R1 \\
\hline Manuscript Type: & Research Paper \\
\hline Date Submitted by the Author: & O8-Sep-2016 \\
\hline Complete List of Authors: & $\begin{array}{l}\text { Wang, Yuekun; University of Manchester, School of Electrical and Electronic } \\
\text { Engineering } \\
\text { Kostakis, Ioannis; Advanced Hall Sensors Ltd } \\
\text { Saeedkia, Daryoosh; TeTechS Inc., } \\
\text { Missous, Mohamed; University of Manchester, School of Electrical and } \\
\text { Electronic Engineering }\end{array}$ \\
\hline Keyword: & $\begin{array}{l}\text { PHOTOCONDUCTING DEVICES, EPITAXIAL GROWTH, III-V } \\
\text { SEMICONDUCTORS, ELECTRO-OPTICAL DEVICES }\end{array}$ \\
\hline &
\end{tabular}

SCHOLARONE

Manuscripts 


\title{
Optimized THz photoconductive devices based on low-temperature grown III-V compound semiconductors incorporating distributed Bragg reflectors
}

\author{
Yuekun Wang ${ }^{1}$, Ioannis Kostakis ${ }^{2}$, Daryoosh Saeedkia ${ }^{3}$ and Mohamed Missous ${ }^{1}$ \\ ${ }^{1}$ School of Electrical and Electronic Engineering, University of Manchester, UK \\ ${ }^{2}$ Advanced Hall Sensors Ltd, Manchester, UK \\ ${ }^{3}$ TeTechS Inc., Waterloo, Ontario, Canada \\ *m.missous@manchester.ac.uk
}

\begin{abstract}
This paper reports work on optimized $\mathrm{THz}$ photoconductive antenna switches based on low temperature grown materials incorporating distributed Bragg reflectors (DBRs). These materials were characterised electrically using Hall Effect, mid-infrared reflectivity measurements and fabricated antennas tested under pulsed excitation in a time domain spectroscopy (TDS) system. It is shown that the inclusion of DBR results in enhanced $\mathrm{THz}$ peak signals by more than twice across the entire operating frequency range. In addition, the devices exhibit significant improvements in the relative $\mathrm{THz}$ power, responsivity and optical to electrical efficiency compared to the reference ones.
\end{abstract}

\section{Introduction}

Nowadays, one of the most widely used methods for generating $\mathrm{THz}$ electromagnetic radiation for $\mathrm{THz}$ time domain spectroscopy (TDS) and $\mathrm{THz}$ two-dimensional imaging applications is through photoconductive antennas [1-4]. In addition, the most promising photoconductive material is low temperature grown GaAs (LT-GaAs) [5]. By using the molecular beam epitaxy (MBE) technique, deep energy levels in the middle of the energy band gap of the semiconductor arise from the formation of point defects during the growth at low temperature, i.e. $\leq 250^{\circ} \mathrm{C}$ and the subsequent precipitation of excess arsenic presents in the material [6]. In this particular case, the LT-GaAs meets all the requirements of photoconductive materials [7, 8]. At room temperature, the band gap of LT-GaAs is $1.42 \mathrm{eV}$, which needs a femtosecond laser operating at $800 \mathrm{~nm}$ to excite it. However, this type of laser, such as Ti-Sapphire, is costly and bulky. In order to build a low cost but still comparable TDS system, alternative materials which have smaller band gaps and that are suitable for use with lasers operating at longer wavelengths (most desired versions are at the $1550 \mathrm{~nm}$ telecommunication wavelength) are required $[9,10]$. THz TDS systems operating at 1550nm based on LT-InGaAs photoconductive antennas have been built and evaluated in [1114]. LT- $\mathrm{In}_{0.53} \mathrm{Ga}_{0.47} \mathrm{As}-\mathrm{In}_{0.52} \mathrm{Al}_{0.48} \mathrm{As}$ which can be operated at $1550 \mathrm{~nm}$ has been reported to show a good response in TDS systems, but the obtained output THz power still does not reach level comparable to that of LT-GaAs [15]. 
By using distributed Bragg reflectors (DBRs), a resonant-optical-cavity photoconductive switch had been reported with significant improvement in performance at $300 \mathrm{GHz}$ [16]. In conventional material systems, the active layers (low temperature grown layers) can absorb only small part of the incident pump laser power and thus the inclusion of DBR layers can reflect most of the unabsorbed radiation back toward the active layers where it has the correct phase to destructively interfere with the residual reflection of the incident beam. In this paper, we report a series of planar aperture and dipole antenna structures fabricated on low temperature grown GaAs and InGaAs-InAlAs (on InP) photoconductors incorporating DBRs. The materials were characterised by performing Hall Effect, mid-infrared reflectivity, and the fabricated devices were evaluated in a TDS system. We demonstrate that this design further improves devices performance in the TDS system with respect to already efficient devices based on III-V semiconductor compounds we reported recently [17].

\section{Sample Descriptions}

The materials used in the photoconductive emitters and detectors are similar to those reported previously for photo-excited $\mathrm{THz}$ photoconductors $[18,19]$. All materials used in this study were grown in a RIBER V100 system using the low temperature MBE growth technique. The active layers for XMBE305 (sample A) and XMBE290 (sample C) were synthetized to absorb light at wavelengths of 800nm and $1550 \mathrm{~nm}$ respectively. The optimized samples with DBR layers labelled as XMBE316 (sample B) and XMBE329 (sample D) were also designed to reflect light at the same wavelengths as XMBE305 and XMBE290. Sample A (LT-GaAs grown to operate at 800nm excitation wavelength) was grown on a semiinsulating GaAs (SI-GaAs) substrate and consisted of a $240 \mathrm{~nm}$ GaAs buffer layer followed by a $50 \mathrm{~nm}$ AlAs layer and a $1 \mu \mathrm{m}$ thickness low temperature grown GaAs layer. The optimized LT-GaAs (sample B) wafer had the same thickness of low temperature grown GaAs layer, but an additional 8 pairs of AlAs/GaAs DBR layers were inserted below the low temperature grown GaAs and above a 100nm GaAs buffer layer. The total thickness of the DBRs was $1.03 \mu \mathrm{m}$. In the case of sample $\mathrm{C}$, the LT- $\mathrm{In}_{0.53} \mathrm{Ga}_{0.47} \mathrm{As}-$ $\mathrm{In}_{0.52} \mathrm{Al}_{0.48} \mathrm{As}$ multi-quantum wells (MQWs) structures, which were synthesised to operate at $1550 \mathrm{~nm}$, were grown on SI-InP substrates, above a 90nm InAlAs buffer layer. The active layers of these samples consist of 50 periods of $12 \mathrm{~nm} \operatorname{In}_{0.53} \mathrm{Ga}_{0.47}$ and $9 \mathrm{~nm} \operatorname{In}_{0.52} \mathrm{Al}_{0.48}$ As super-lattice structure and an $\operatorname{In}_{0.53} \mathrm{Ga}_{0.47}$ cap layer which were also grown at low temperature. All active layers were uniformly doped with Be atoms with a doping concentration of $2 \times 10^{18} \mathrm{~cm}^{-3}$. The optimized LT-InGaAs/InAlAs (sample D) wafer contained 50 periods of $12 \mathrm{~nm}$ InGaAs and $12 \mathrm{~nm}$ InAlAs super-lattice structure with the same doping concentration as active layers in sample C. The 8 pairs of InGaAs-InAlAs DBR layers with a total 
thickness of $1.65 \mu \mathrm{m}$ were sandwiched by the active layers and InP substrate. The epitaxial layers of all samples investigate in this work are shown in Fig.1.

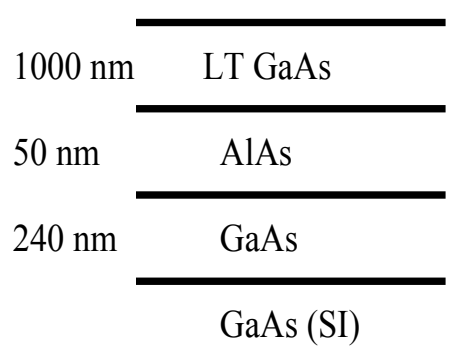

$a$

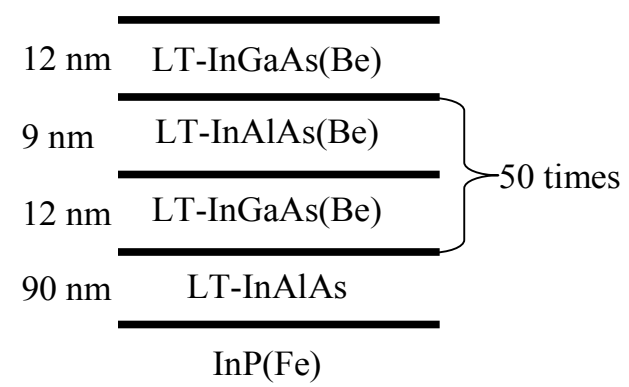

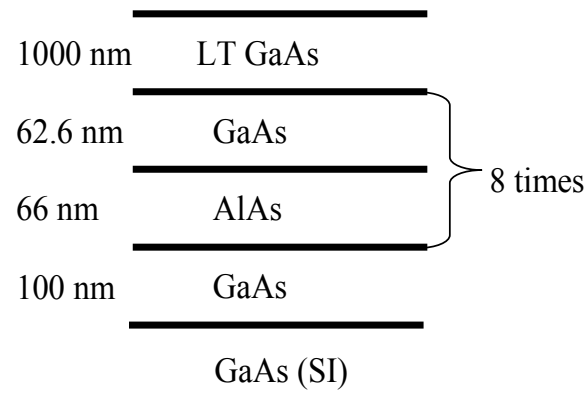

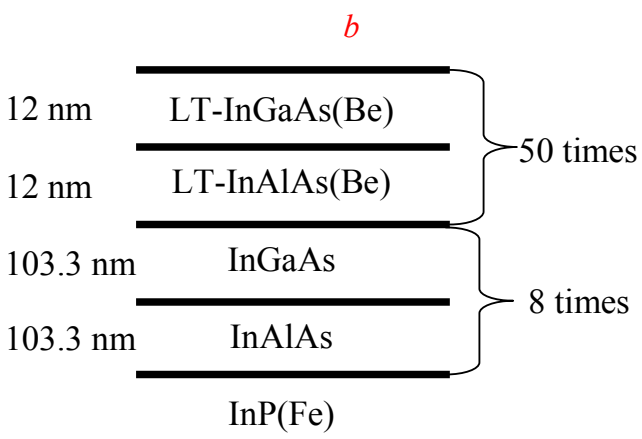

Fig. 1 Physical structures along with the layer thicknesses

a LT-GaAs (sample A)

b LT-GaAs with DBRs (sample B)

c LT-InGaAs-InAlAs (sample C)

d LT-InGaAs-InAlAs with DBRs (sample D)

In order to improve the electro-optical characteristics of the samples, annealing at specific temperatures $\left(600^{\circ} \mathrm{C}\right.$ for LT-GaAs and $580^{\circ} \mathrm{C}$ for LT-InGaAs/InAlAs) was necessary [20]. All samples were annealed for 10 minutes and were covered by high purity SI GaAs substrates to prevent arsenic loss from the sample surface. This post growth annealing was performed using a Rapid Thermal Annealer (RTA) in a nitrogen atmosphere. RTA allowed the formation of precipitates form the excess arsenic incorporated as point defects [19]. To investigate the effect of the DBR layers, materials were fabricated as antennas in the same epitaxial structures (aperture for emitters and dipole for emitters or detectors). The choice of dipole and aperture geometries as antenna structures was based on a previous study [21]. In that study, the radiation efficiencies of resonant dipole antennas and wide-band aperture antennas were compared and found to be adequate in order to characterise the performance of the photoconductor materials. Fig. 2 illustrates the geometries and dimensions of the aperture and dipole antennas. The aperture structures were designed to have $500 \mu \mathrm{m}$ width with a separation (gap) between the electrodes of 
$400 \mu \mathrm{m}$. The dipole antennas had $20 \mu \mathrm{m}$ arm's length and the gap between the two arms was $5 \mu \mathrm{m}$. All the antenna structures were fabricated on top of the photoconductive materials by standard i-line photolithography techniques. Both AuGe based alloys and Ti/Au metals are usually used as the contacts for photoconductive THz antennas[22-24], Ti/Au deposition was chosen in this work to define the antenna region of the photoconductors. After the metallization step, the samples were sintered at $250{ }^{\circ} \mathrm{C}$ to enable good adhesion of the contacts.

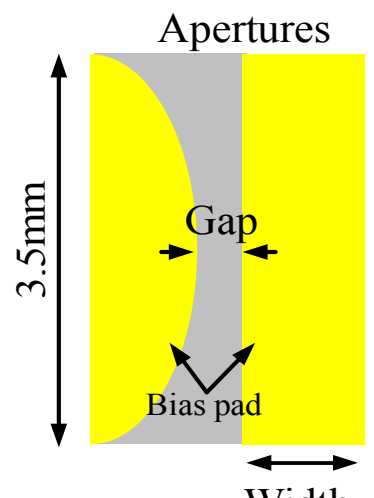

Width

$a$

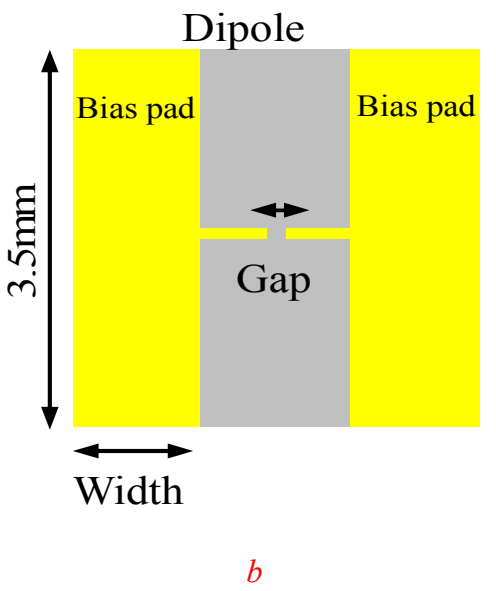

Fig. 2 Antenna structures designed for THz emitters and detectors a aperture geometry

b dipole geometry

\section{Material Characterisations}

\subsection{Transport properties}

The transport properties of the samples were studied using Hall Effect measurements. The measurements were performed at room temperature on Van Der Pauw structures using a customised set-up consisting of a Keithley current and voltage source measuring units and permanent magnets. Cloverleaf geometries were patterned using standard photolithography technique and wet etching methods with four pieces of Indium (In) alloys used to form the Ohmic contacts.

Both samples A and B were annealed at $600^{\circ} \mathrm{C}$. Sample A exhibited a mobility of $2400 \mathrm{~cm}^{2} / \mathrm{Vs}$, carrier concentration of $2.8 \times 10^{6} \mathrm{~cm}^{-2}$, and a resultant sheet resistance of $9.3 \times 10^{8} \Omega / \mathrm{sq}$ [18]. Sample B exhibited a mobility of $2200 \mathrm{~cm}^{2} / \mathrm{Vs}$, carrier concentration of $2.9 \times 10^{6} \mathrm{~cm}^{-2}$, and a sheet resistance of 9.7 $\mathrm{x} 10^{8} \Omega /$ sq. Both samples $\mathrm{C}$ and $\mathrm{D}$ were annealed at $580^{\circ} \mathrm{C}$. Because of the high Be-doping throughout the active layers and the less than optimal compensation of the n-type defects in the LT-InGaAs, the mobilities 
were much lower at $425 \mathrm{~cm}^{2} / \mathrm{Vs}$ [15] and $410 \mathrm{~cm}^{2} / \mathrm{Vs}$ for samples C and D respectively. The carrier concentrations were $2.0 \times 10^{9} \mathrm{~cm}^{-2}$ and $1.9 \times 10^{9} \mathrm{~cm}^{-2}$ for samples $\mathrm{C}$ and D. The corresponding sheet resistances of samples $\mathrm{C}$ and $\mathrm{D}$ were $7.2 \times 10^{6} \Omega / \mathrm{sq}$ and $7.9 \times 10^{6} \Omega /$ sq respectively. Samples $\mathrm{C}$ and $\mathrm{D}$ exhibited one order of magnitude higher sheet resistance than the best LT-InGaAs-InAlAs reported in the literature [17].

According to the data shown above, the transport properties of the normal samples and their DBR counterparts are comparable, thus a conclusion can be reached that the DBR layers do not affect the electrical performance of the active layers.

\subsection{Mid-Infrared Reflectivity Measurements}

In order to study the optical effect of the DBR layers, reflection measurements were carried out at room temperature using an Ocean Optics LS1 tungsten halogen light source. The detector for the LTInGaAs-InAlAs structures was an Ocean Optics NIR256-2, while an Ocean Optics S2000 CCD detector was used for the LT-GaAs devices. Sample B was split into three pieces (As grown, B1 and B2) and prepared using a wet etching method. Samples were etched to two different depths. The first one terminated marginally above the DBR layers while the second was etched into the DBR layers. The depths of sample B1 and B2 were 960nm and 1020nm respectively. Sample D was prepared in a similar manner providing depths of $1070 \mathrm{~nm}$ and $1270 \mathrm{~nm}$ for sample D1 and D2 respectively. In order to obtain a direct response of the DBRs, the reflectance measurements were normalised using the as grown samples (B and D). The measured results are shown in Fig. 3 (a) and (b).
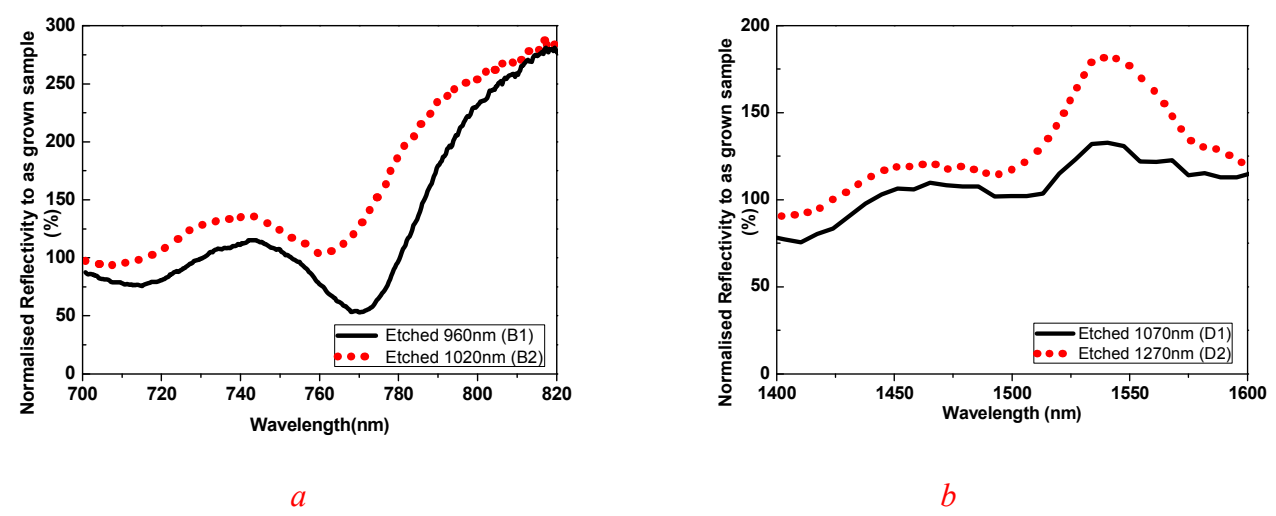

Fig. 3 Normalised reflectivity

a LT-GaAs samples with DBRs: B1(black solid line) and B2 (red dash line)

b LT-InGaAs/InAlAs samples with DBRs: D1(black solid line) and D2 (red dash line) 
The black solid line in Fig. 3 can be considered as the contribution that includes the response of both the active and DBR layers. Since the as grown sample was used as the reference and compared with the etched sample (which had a majority of its active layers removed), the normalised reflectivity is shown to be higher than the reference at all wavelengths. This is a consequence of the active layers absorbing more radiation in the reference sample and this is more evident with increasing etch depth (red dash lines in Fig. 3). The highest normalised reflectivity is approximately twice the as grown reference sample obtained at $800 \mathrm{~nm}$ for sample B.

\section{Antenna Characterisation}

The fabricated antennas were evaluated as emitters and detectors in a TDS system under pulsed operations at room temperature in an un-purged environment. In this setup, a Mai Tai pulse laser from Spectra Physics operating at 800nm wavelengths was used for samples A and B, and a FemtoFiber FFS short pulse laser from Toptica Photonics AG operation at 1550nm was used for sample D. A beam splitter was used to separate the output pulse from the laser source into the pump beam and the probe beam. The pump beam was focused onto the surface of the emitter while the probe beam was delayed and focused onto the detector. High resistivity hyper-hemispherical Si lenses were attached at the back side of the antennas in order to collimate and focus the $\mathrm{THz}$ radiation. In the $1550 \mathrm{~nm}$ operation system, the laser beam can be easily coupled to the photoconductive antenna by using optical fibers thus making the system compact and portable. In the case of $800 \mathrm{~nm}$ operating system, the optical pulse duration was $100 \mathrm{fs}$, the pulse repetition rate was $80 \mathrm{MHz}$ while the pump and the probe beam average power were $25 \mathrm{~mW}$ and $10 \mathrm{~mW}$ respectively. In the case of $1550 \mathrm{~nm}$ system, the optical pulse duration was $100 \mathrm{fs}$, and the pulse repetition rate was $86 \mathrm{MHz}$ while the pump and the probe beam average powers were $14.5 \mathrm{~mW}$ and $14 \mathrm{~mW}$ respectively.

\subsection{LT-GaAs Antennas}

To investigate the photoconductor antenna with DBRs, two tests were performed. The first set of measurement used sample A as the emitter while the second one used sample B as the emitter. Throughout the measurements, emitters with $400 \mu \mathrm{m}$ gap were under a bias of $50 \mathrm{~V}$ and the reference receiver device was a dipole antenna fabricated on material XMBE305 (sample A). Fig.4 depicts the comparison of emitted THz pulses and Fourier power spectrums using samples with and without DBRs as emitter (the sample without DBRs was used as detector for both measurements). 


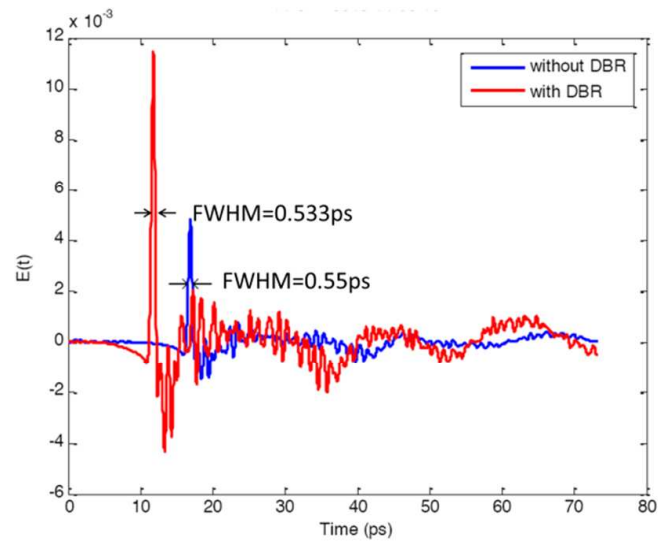

$a$

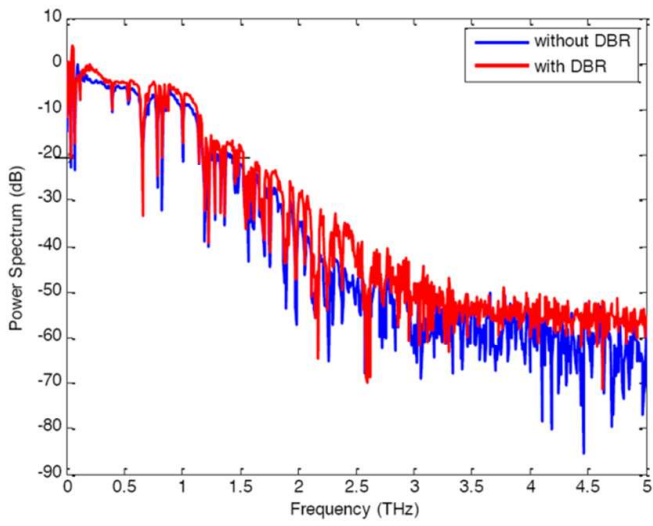

$b$

Fig. 4 Devices evaluation in the TDS system at 800nm pulse excitation

a THz pulses generated from aperture antennas made on sample A ((LT-GaAs: blue line) and sample B (LT-GaAs with DBRs: red line) and detected by dipole antenna made on sample A

b normalized Fourier transform power spectrums emitted from aperture antennas made on sample A (LT-GaAs: blue line) and sample B (LT-GaAs with DBRs: red line) and detected by a dipole antenna made on the sample A

The full width half maximum (FWHM) was 0.533 ps indicating a reasonably fast switching speed of the photoconductor. The $20 \mathrm{~dB}$ bandwidth using sample B as emitter indicated a spectral extent of $1.44 \mathrm{THz}$ and the dynamic range extended up to $3.5 \mathrm{THz}$ with power to noise ratio of more than $60 \mathrm{~dB}$ (noise floor). When sample A was used as emitter, the FWHM was $0.55 \mathrm{ps}$, and the $20 \mathrm{~dB}$ bandwidth was $1.32 \mathrm{THz}$ with a $4 \mathrm{THz}$ for $60 \mathrm{~dB}$ noise floor. The THz peak signal from sample B is $11.5 \mathrm{nA}$, this is more than twice that of the peak signal without DBRs. By taking into account the incident THz electric field and the measured current, the relative $\mathrm{THz}$ power of the emitter can be estimated in arbitrary units. Therefore, the relative magnitudes of the $\mathrm{THz}$ power of sample $\mathrm{A}$ and $\mathrm{B}$ are 2050 and 6900 , with responsivities of $0.57 \mathrm{nA} / \mathrm{mW}$ and $1.15 \mathrm{nA} / \mathrm{mW}$ respectively. The optical to electrical efficiency of the sample was determined by the photoconductive power and pump power, the results of samples A and B were $1.47 \%$ and $3.6 \%$ respectively. Thus, in comparing samples A and B, we conclude that the DBRs under LT-GaAs does not affect the switching speed but rather results in enhancing the $\mathrm{THz}$ peak signal, the relative magnitude of the $\mathrm{THz}$ power, and the optical to electrical efficiency by more than twice as well increasing the transmitter photocurrent.

\subsection{LT-InGaAs-InAlAs MQWs Antennas}

In the case of the LT-InGaAs-InAlAs MQWs samples, the measurement set up was similar to LTGaAs. Sample D was fabricated into a dipole antenna for both emission and detection and the emitter was bias at $3 \mathrm{~V}$. 


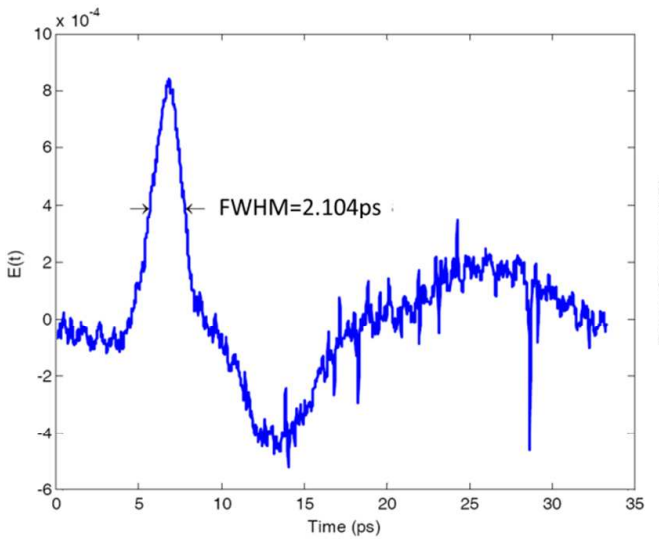

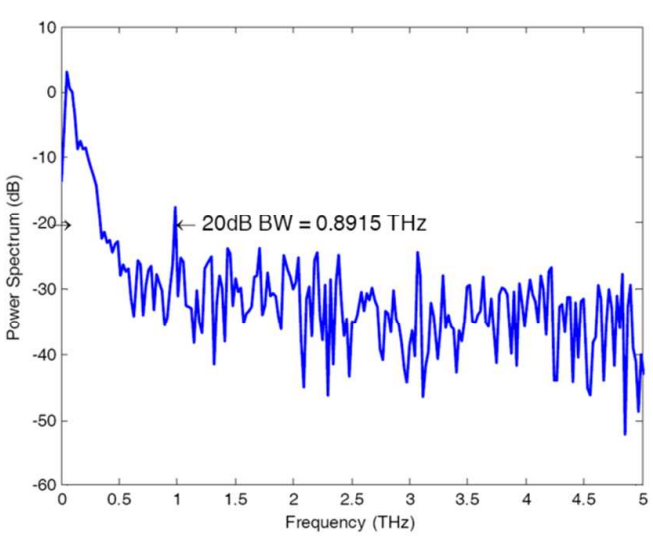

$b$

Fig. 5 Devices evaluation in the TDS system at $1.55 \mu$ m pulse excitation a THz pulse generated and detected by dipole antennas made on sample D (LT-InGaAs with DBRs) b normalized Fourier transform power spectrum emitted and detected by dipole antennas made on sample D (LT-InGaAs with DBRs)

The LT-InGaAs-InAlAs MQWs sample had already been evaluated and reported in [15, 19]. The photoconductive switching speed of sample D was not as fast as reported in our previous report and the bandwidth was also narrower. These results indicate a poor bandwidth compared to previous systems reported by our group or compared to commercial available systems. This can be explained by the doping profile used (uniform doping throughout the active layers) and also due to the antenna geometry chosen. Nevertheless, with the inclusion of the DBRs, the relative magnitude of the THz power of sample D was 57 with a responsivity of $0.086 \mathrm{nA} / \mathrm{mW}$, compared with similar material systems [15] that showed relative magnitude of the $\mathrm{THz}$ power of 0.83 and a responsivity of $0.008 \mathrm{nA} / \mathrm{mW}$. These results show clearly the importance of the incorporation of DBR layers in increasing the optical to electrical efficiency of sample $\mathrm{D}$ which rose to $1.14 \%$, and is approximately double the efficiency of similar material system.

\section{Conclusions}

This work reports on a systematic study showing enhanced and excellent performance of photoconductive switches integrated with DBR layers in GaAs and InGaAs-InAlAs technologies. The designed materials can be fabricated as $\mathrm{THz}$ emitters and detectors operating at room temperature at both $800 \mathrm{~nm}$ and telecom communication wavelength of $1550 \mathrm{~nm}$. The DBRs result in a marked enhancement of the THz peak signal by more than twice, an increase of the transmitter photocurrent and a doubling of the optical to electrical efficiency. By coupling DBRs with active layers, the performances of photoconductive switches show better responses and further allow for higher quality $\mathrm{THz}$ measurements to be made. 


\section{Acknowledgments}

We are grateful for the UK's EPSRC support for part of this work. One of us (Yuekun Wang) is also grateful to China Scholarship Council for financial support.

\section{References}

[1] E. Brown, 'THz generation by photomixing in ultrafast photoconductors' International Journal of High Speed Electronics and Systems, vol. 13, pp. 497-545, 2003.

[2] D. Saeedkia, 'Handbook of terahertz technology for imaging, sensing and communications' Elsevier, 2013.

[3] G. Carpintero, E. Garcia-Munoz, H. Hartnagel, S. Preu, and A. Räisänen, 'Semiconductor TeraHertz Technology: Devices and Systems at Room Temperature Operation' John Wiley \& Sons, 2015.

[4] D. Saeedkia and S. Safavi-Naeini, 'A comprehensive model for photomixing in ultrafast photoconductors' IEEE photonics technology letters, vol. 18, pp. 1457-1459, 2006.

[5] P. Kordos, F. Ruders, M. Marso, and A. Forster, 'Properties of LT GaAs for photomixing up to $\mathrm{THz}$ frequencies' Optoelectronic and Microelectronic Materials and Devices Proceedings (Institute of Thin Film and Ion Technology, Research Centre Julich, Germany, 1996), pp. 71-74, 1996.

[6] M. Luysberg, H. Sohn, A. Prasad, P. Specht, Z. Liliental-Weber, E. Weber, et al., 'Effects of the growth temperature and As/Ga flux ratio on the incorporation of excess As into low temperature grown GaAs' Journal of applied physics, vol. 83, pp. 561-566, 1998.

[7] G. Martin, 'Optical assessment of the main electron trap in bulk semi-insulating GaAs' Applied Physics Letters, vol. 39, pp. 747-748, 1981.

[8] G. Martin, J. Farges, G. Jacob, J. Hallais, and G. Poiblaud, 'Compensation mechanisms in GaAs' Journal of Applied Physics, vol. 51, pp. 2840-2852, 1980.

[9] R. Takahashi, Y. Kawamura, and H. Iwamura, 'Ultrafast $1.55 \mu \mathrm{m}$ all-optical switching using low temperature-grown multiple quantum wells' Applied physics letters, vol. 68, pp. 153-155, 1996.

[10] K. Biermann, D. Nickel, K. Reimann, M. Woerner, T. Elsaesser, and H. Künzel, 'Ultrafast optical nonlinearity of low-temperature-grown GaInAs/AlInAs quantum wells at wavelengths around 1.55 um' Applied physics letters, vol. 80, pp. 1936-1938, 2002.

[11] B. Sartorius, H. Roehle, H. KŘnzel, M. Schlak, D. Stanze, H. Venghaus, et al., 'All-fiber terahertz time-domain spectrometer operating at $1.5 \mu \mathrm{m}$ telecom wavelengths' Optics Express, vol. 16, pp. 9565-9570, 2008.

[12] N. Vieweg, F. Rettich, A. Deninger, H. Roehle, R. Dietz, T. Göbel, et al., 'Terahertz-time domain spectrometer with $90 \mathrm{~dB}$ peak dynamic range' Journal of Infrared, Millimeter, and Terahertz Waves, vol. 35, pp. 823-832, 2014.

[13] R. Wilk, M. Mikulics, K. Biermann, H. Künzel, I. Z. Kozma, R. Holzwarth, et al., 'THz timedomain spectrometer based on LT-InGaAs photoconductive antennas exited by a $1.55 \mu \mathrm{m}$ fibre laser' Conference on Lasers and Electro-Optics, 2007.

[14] B. Sartorius, H. Künzel, K. Biermann, J. Böttcher, H. Roehle, R. Wilk, et al., 'THz Photoconductive Antennas for $1.55 \mu \mathrm{m}$ Telecom Wavelengths' Optical Terahertz Science and Technology, 2007.

[15] I. Kostakis, D. Saeedkia, and M. Missous, 'Terahertz generation and detection using low temperature grown InGaAs-InAlAs photoconductive antennas at 1.55 pulse excitation' IEEE Transactions on Terahertz Science and Technology, vol. 2, pp. 617-622, 2012. 
[16] Z. Taylor, E. Brown, J. Bjarnason, M. Hanson, and A. Gossard, 'Resonant-optical-cavity photoconductive switch with $0.5 \%$ conversion efficiency and $1.0 \mathrm{~W}$ peak power' Optics letters, vol. 31, pp. 1729-1731, 2006.

[17] I. Kostakis, D. Saeedkia, and M. Missous, 'Efficient terahertz devices based on III-V semiconductor photoconductors' IET Optoelectronics, vol. 8, pp. 33-39, 2014.

[18] M. Missous, I. Kostakis, and D. Saeedkia, 'Long wavelength low temperature grown GaAs and InP-based Terahertz photoconductors devices' IEEE Sensors Journal, vol. 13, pp. 63-71, 2013.

[19] I. Kostakis and M. Missous, 'Optimization and temperature dependence characteristics of low temperature $\mathrm{In}_{0.3} \mathrm{Ga}_{0.7} \mathrm{As}$ and $\mathrm{In}_{0.53} \mathrm{Ga}_{0.47} \mathrm{As}-\mathrm{In}_{0.52} \mathrm{Al}_{0.48} \mathrm{As}$ semiconductor terahertz photoconductors' AIP Advances, vol. 3, p. 092131, 2013.

[20] I. S. Gregory, C. Baker, W. Tribe, M. Evans, H. E. Beere, E. H. Linfield, et al., 'High resistivity annealed low-temperature GaAs with 100 fs lifetimes' Applied physics letters, vol. 83, pp. 41994201, 2003.

[21] D. Saeedkia, 'Resonantly enhanced terahertz power spectrum in terahertz photoconductive antennas' 34th International Conference on Infrared, Millimeter, and Terahertz Waves, 2009.

[22] I. C. Mayorga, M. Mikulics, A. Schmitz, P. Van der Wal, R. Gusten, M. Marso, et al., 'An optimization of terahertz local oscillators based on LT-GaAs technology' SPIE Millimeter and Submillimeter Dtectors for Astronomu II, pp. 537-547, 2004.

[23] N. Vieweg, M. Mikulics, M. Scheller, K. Ezdi, R. Wilk, H.-W. Hübers, et al., 'Impact of the contact metallization on the performance of photoconductive THz antennas' Optics express, vol. 16, pp. 19695-19705, 2008.

[24] M. Mikulics, M. Marso, S. Wu, A. Fox, M. Lepsa, D. Grutzmacher, et al., 'Sensitivity Enhancement of Metal-Semiconductor-Metal Photodetectors on Low-Temperature-Grown GaAs Using Alloyed Contacts' IEEE Photonics Technology Letters, vol. 20, pp. 1054-1056, 2008. 